\title{
Influencia del sexo y la lengua materna en el rendimiento académico
}

\section{Influence of Sex and Mother Tongue on Academic Performance}

Francisco J. Olmedo Ruiz*

Universidad de Granada, Granada, España

ORCID: https://orcid.org/0000-0002-0180-9288

Francisco Mateos Claros

Universidad de Granada, Granada, España

ORCID: https://orcid.org/0000-0002-6596-9803

María J. Latorre Medina

Universidad de Granada, Granada, España

ORCID: https://orcid.org/0000-0003-2237-9341

\section{Macarena Esteban Ibáñez}

Universidad Pablo de Olavide, Sevilla, España ORCID: https://orcid.org/0000-0002-2246-4784

Recibido 28-08-19 Revisado 12-10-19 Aprobado 10-01-19 En línea 20-01-20

*Correspondencia

Email: folmedoruiz@gmail.com
Citar como:

Olmedo, F., Mateos, F., Latorre, M., \& Esteban, M. (2020) Influencia del sexo y la lengua materna en el rendimiento académico. Propósitos y Representaciones, 8(1), e382. doi: http://dx.doi.org/10.20511/pyr2020.v8n1.382 


\section{Resumen}

Este trabajo se centra en conocer las diferencias en el rendimiento académico de los alumnos de Educación Infantil según el sexo y la lengua materna. Para ello, y partiendo de los distintos escenarios que nos presenta la multiculturalidad, hemos tenido en cuenta las peculiaridades de esta diversidad cultural reflejadas en el aula, sin olvidar que nos encontramos en un contexto en el que coexiste una lengua oficial y un dialecto cultural. La muestra estuvo integrada por 489 alumnos de Educación Infantil de 12 centros educativos públicos y 3 centros concertados, de edades comprendidas entre 5 y 6 años $(\mathrm{M}=5.23$; DT $=.506)$; de estos el $51.4 \%$ eran niños $(n=249)$ y el $48.6 \%$ niñas $(n=235)$, de los cuales el $37.7 \%$ tenían como lengua materna familiar el castellano $(\mathrm{n}=183)$ y el $62.3 \%$ el darija $(\mathrm{n}=302)$. Se utilizó el instrumento multidimensional de competencias básicas en Educación Infantil (Mateos y Olmedo, 2018). Los resultados de los análisis ANOVA y regresión Logística Binaria evidenciaron que la lengua materna es la principal causa de las diferencias de estos niños, afectando negativamente a aquellos que tienen lengua materna castellana, quedando claramente definido que la principal debilidad se muestra en las áreas de la expresión oral, la expresión escrita y la conciencia fonética.

Palabras claves: Educación pre-primaria; Rendimiento académico; Diversidad lingüística; Sexo.

\section{Summary}

This work focuses on knowing the differences in the academic performance of children's education students according to sex and mother tongue. To this end, and starting from the different scenarios presented by multiculturality, we have taken into account the peculiarities of this cultural diversity reflected in the classroom. On the basis that we are in a context in which an official language and a cultural dialect coexist. The sample consisted of 489 children's education students from 12 public educational establishments and 3 concerted centres, aged 5 to $6(\mathrm{M}=5.23$; DT $=506)$, of which 51.4 percent were boys $(\mathrm{n}=249)$ and 48.6 percent girls $(n=235)$ of which $37.7 \%$ had Spanish as their mother tongue $(n=183)$ and $62.3 \%$ Darija $(\mathrm{n}=302)$. The multidimensional instrument of basic skills in Child Education was used (Mateos y Olmedo, 2018). The results of ANOVA analysis and binary logistic regression showed that the mother tongue is the main cause of these children's differences, negatively affecting those who have mother tongue Castilian, being clearly defined that the main weakness is shown in the areas of oral expression, written expression and phonetic consciousness.

Keywords: Pre-Primary Education; Academic Performance; Language Diversity; Sex.

\section{Introducción}

El desarrollo de habilidades cognitivas, lingüísticas, afectivas y sociales en el periodo infantil comprende una serie de cambios evolutivos influenciados por múltiples factores. En esta etapa, los procesos del aprendizaje infantil instauran una relación interna para comprender el mundo en el que intervienen la adquisición del lenguaje (Chomsky, 1968), el desarrollo motriz y el progreso social y afectivo (Bowlby, 1993) y una relación de carácter externo que se relaciona con el contexto y sus características (Vygotsky, 1962; Wild, 2011). De ahí, que en el inicio de la educación formal se muestren distintos factores que condicionan el progreso educativo de estos alumnos antes de finalizar el periodo infantil (Olmedo, 2017). De hecho, en este ámbito, el sexo, la cultura, la familia, etc., se presentan como unas de las principales dimensiones que pueden influir en este progreso (Goñi, Ros y Fernández, 2018).

Partiendo de la importancia del lenguaje, y con independencia a los distintos estudios que corroboran que las niñas tienen un mejor rendimiento esta área (Harris, 1977; Kimura, 1999; Maccoby y Jacklins, 1974; Moss y Brown, 1979; Smolak, 1986), este se convierte en el 
principal vehículo para el avance de las distintas dimensiones relacionadas con el desarrollo evolutivo, además de un recurso vital para la interacción social (Piaget, citado por Maier, 2000; Méndez, Barrientos, Macías y Peña, 2006). En consecuencia, se deben de considerar como las carencias lingüísticas asociadas a factores externos -familia y cultura- pueden limitar el progreso comunicativo en este periodo (Latorre, Mateos, Olmedo y Esteban, 2018). Por todo esto, debemos de tener en cuenta los factores que intervienen en este proceso, dado que el área comunicativa representa uno de los avances más significativos de esta etapa en el que es imprescindible separar la exposición del desarrollo evolutivo en varias áreas sin descartar el proceso global. Stanley y Greenspan (2000) destacan cómo en este desarrollo incide la capacidad de separar las palabras de los objetos, la creación del símbolo en diferentes niveles, las interacciones del lenguaje no verbal con el sonido, la regularidad del lenguaje fonético, etc. en la evolución del lenguaje. En consecuencia, en este periodo las carencias de estas dimensiones pueden mermar la evolución del lenguaje afectando de forma directa a la consecución de la lectoescritura (Defior, 2014).

Los contextos diversos que representan el panorama social actual bajo el proceso dominante de la globalización provocan un constante intercambio cultural, que tienen su mayor expresión en la coexistencia de distintos códigos lingüísticos. De ahí, la influencia directa del uso comunicativo en el ámbito familiar (Bartolomé, 2017; González y Martín, 2017; Moerk, 1975; Sousa, 2014), aunque para Gleitman, Newport y Gleitman (1984) este asociada a influencias superficiales. Estos contactos lingüísticos, en ocasiones adheridos a una cultura y a reproducciones vocálicas distintas (Abu-Shams, 2011), repercuten en los aprendizajes escolares (Latorre et al., 2018). Por ello, el dominio lingüístico en edades tempranas es un elemento reductor de las desigualdades que enfatiza el valor de la enseñanza de la lengua en la etapa de Educación Infantil (Palau, 2005; Turner y Cross, 2016), sin obviar su importancia y la influencia en el mismo de factores externos. Una situación que resalta la influencia cultural en los estilos lingüísticos que puede interferir en los patrones de interacción (Goleman, 1996).

La integración en nuevos ámbitos distintos del familiar hace que la competencia lingüística se convierta en un elemento vital para estos nuevos ámbitos de interacción. Por ello, el espacio escolar se presenta como uno de los principales ámbitos a la hora de potenciar las relaciones intergrupales y la interacción en contextos interculturales (Sanhueza y Cardona, 2009). Es imprescindible encontrar un punto de encuentro entre el modelo de aprendizaje que se cultiva en el núcleo familiar y el ofrecido por la institución escolar (Rodríguez, 2017), teniendo presente que en contextos donde existe contacto entre distintas lenguas, presidido por el uso de un código lingüístico distinto del que vehicula el currículo escolar y claramente dinamizado por la cultura, este puede interferir en los procesos de aprendizajes (Mora, 2013; Tejedor y García-Valcárcel, 2006).

Partiendo de la base de que nos encontramos en un periodo de transición educativa, el progreso educativo se convierte en elemento básico para el desarrollo evolutivo. De acuerdo con Gómez, Oviedo y Martínez (2011), el rendimiento académico es el resultado de una serie de factores que actúan en, y desde, la persona que aprende. En cambio, Marjoribanks (2005) defiende que el entorno es el que predispone el nivel educativo que este obtendrá, asociados a ambientes personales, familiares y sociales. Si a esto le añadimos la heterogeneidad que muestran ciertas particularidades definidas por el sexo, la identidad cultural, los usos lingüísticos y las amistades (Sanhueza y Carmona, 2009), el rendimiento académico muestra una variedad muy amplia de factores que interfieren en su avance que lo convierte en uno de los temas con mayor relevancia de la investigación educativa (Lamas, 2015).

De ahí que la seña de identidad de las diferencias multiculturales tenga su máxima representación en el lenguaje, siendo este unas de las causas principales de las diferencias académicas en la Educación Infantil (Olmedo, 2017). En consecuencia, el área comunicativa se presenta como un referente para la interculturalidad, al mismo tiempo que un elemento diferenciador de la identidad de los grupos (Sanhueza y Carmona, 2009). Estas divergencias 
lingüísticas pueden limitar la relación con el otro repercutiendo de forma negativa en los procesos de integración y socialización en estas edades. En este campo, distintas investigaciones han confirmado que las diferencias entre el uso funcional de la lengua en el hogar y en la escuela pueden interferir en el progreso educativo por la impregnación de unos patrones lingüísticos que difieren del escolar (Gay, 2000; Harris, Kamhi, y Pollock, 2001; Mateos, Olmedo, Esteban y Amador, 2019).

Asumiendo las distintas peculiaridades que representan los contextos multiculturales, y centrándonos en el ámbito educativo, presentamos los objetivos de esta investigación:

1. Identificar cuáles son las diferencias en el rendimiento lingüístico según el sexo y la lengua materna.

2. Conocer el principal elemento que explica las diferencias en el rendimiento comunicativo de estos alumnos.

3. Averiguar cuáles son las principales áreas comunicativas que inciden en el desarrollo lingüístico de estos alumnos.

\section{Método}

Para el diseño de la investigación nos hemos centrado en una metodología descriptiva, de carácter transversal y cuantitativo. El método utilizado para la elección de los sujetos del estudio se ha basado en la aplicación de un muestreo aleatorio, partiendo de las variables lengua materna (castellano; darija) y sexo (niño; niña) a la hora de hacer los estratos.

\section{Participantes}

La muestra seleccionada para este trabajo estuvo centrada en el alumnado de Educación Infantil de segundo ciclo (5 años) de distintos centros educativos (públicos y concertados) de Ceuta con la intención de plasmar las peculiaridades de este espacio multicultural. Para la elección de los sujetos del estudio se aplicó un muestreo aleatorio teniendo en cuenta la lengua materna de los alumnos castellanoparlantes y darijoparlantes. La muestra estuvo integrada por 489 escolares $\left(\mathrm{N}=3721 ; \mathrm{Z}_{\mathrm{a}}^{2}=1.96^{2}\right.$ seguridad del 95\%; proporción esperada $5 \%=0.05 ; \mathrm{q}=1-\mathrm{p}=0.95 ; \mathrm{d}=$ precisión un $2 \%$ ) de 12 centros educativos públicos y 3 centros concertados. La edad media de estos alumnos es de 5.23 (DT= .506), de estos el $51.4 \%$ eran niños $(n=249)$ y el $48.6 \%$ niñas $(\mathrm{n}=235)$, de los cuales el $37.7 \%$ tenían como lengua materna familiar el castellano $(\mathrm{n}=183)$ y el $62.3 \%$ el darija $(\mathrm{n}=302)$.

\section{Instrumentos}

El instrumento utilizado fue de elaboración propia (Mateos y Olmedo, 2018). Se realizó a partir de la revisión de distintas fuentes bibliográficas relacionadas con el desarrollo evolutivo, el rendimiento académico y competencias básicas (Piaget, 1988; Vygotsky, 1996; Wild, 2011). Seguidamente, comparamos distintos instrumentos estandarizados como el WISC, Merrill Palmer R (Roid y Sampers, 2011), pruebas pedagógicas graduadas, etc., que nos aportaron más información a la hora de seleccionar los ítems de este instrumento. El instrumento consta de 55 ítems, que se cumplimentan a través de una escala de Likert de 4 categorías ( $1=$ nunca, $2=\mathrm{a}$ veces, $3=$ a menudo, $4=$ siempre), donde se recoge el grado de adquisición de aspectos competenciales básicos de esta etapa. Las distintas variables comprenden: el área de comunicación y lenguaje (comprensión oral, expresión oral, expresión escrita, conciencia fonética, y lenguaje matemático) que cuenta con 30 ítems; el área de habilidades sociales (desarrollo emocional, la actitud ante los compañeros, la actitud ante el trabajo, ante el juego y hábitos) en la que se agrupan 19 ítems; y, finalmente, el área motriz con 11 ítems.

Para medir la fiabilidad se aplicó el cálculo estadístico Alpha de Cronbach, una de las pruebas más utilizadas en investigaciones psicométricas (Maroco \& García, 2013). Mostrando 
en el caso del rendimiento académico una consistencia interna total de .959 muy aceptable (escala comunicativa .960; de habilidades sociales .791; de motricidad .871). La validez de constructo se realizó a través de un análisis factorial exploratorio con un valor de KMO .959 y .000 en la prueba de Bartlett .000.

\section{Procedimiento}

Antes de iniciar la recogida de datos obtuvimos el consentimiento informado por parte de la Delegación del Ministerio de Educación y Formación Profesional de la Ciudad Autónoma de Ceuta. A continuación, el instrumento de rendimiento académico fue repartido en los centros de Educación Infantil (5 años) seleccionados para que fueran cumplimentados por los docentes. Con anterioridad a su cumplimentación se hicieron aclaraciones que pudieran surgir a la hora de contestar los ítems, además de ser informados de su carácter anónimo, confidencial y voluntario. La fecha establecida para la entrega se determinó para el mes de mayo, con la intención de acotar al máximo el curso escolar. El tiempo estimado para el instrumento osciló entre 15 y 25 minutos por alumno. Finalmente, en la recolección se verificó que todos los ítems estuvieran contestados.

\section{Análisis de datos}

Para analizar los datos obtenidos a través del instrumento se ha utilizado el paquete estadístico SPSS 22.0. Inicialmente, la estadística descriptiva nos permitió conocer las características de la muestra. Para contrastar las diferencias significativas en el rendimiento de estos alumnos los estadísticos de contraste (ANOVA) facilitaron una visión sobre la relación o divergencia entre los resultados esperados y observados (Glass y Stanley, 1980). Seguidamente, se aplicó un análisis de regresión logística binario como técnica multivariante, tomando como variables la lengua materna castellana y lengua materna darija. La intención principal fue valorar si las variables que se han citado presentaban una convicción logística óptima, partiendo de la base de su condición de variable dicotómica e intentando valorar si la combinación seleccionada puede ayudar a pronosticar mejor el rendimiento académico en el área comunicativa en función de la lengua materna (castellano y darija). Inicialmente, se aplicó la prueba de ajuste ómnibus, valorando que las variables ya combinadas en el análisis logístico se distribuyen con un $p$-valor $<.05$ que nos permita estimar que presentan gran influencia en la mejor predicción la lengua materna darija en comparación con la lengua materna castellana. Igualmente, la prueba de hosmer y lemeshow (>.05) verificó la idoneidad de la prueba logística y la contingencia nos permitió observar si los valores se aproximan a los esperados y comparar si existe mayor aproximación entre uno de los dos grupos con la finalidad de continuar verificando la mejor predicción de una de las dos variables dependientes en base a las dimensiones seleccionadas

\section{Resultados}

Para conocer la distribución de las variables según su comparación vamos a presentar las tablas $1,2,3$, donde podemos observar las medias y significación de las dimensiones seleccionadas.

Teniendo en cuenta el primer objetivo, los resultados de la tabla 1 muestran la existencia de diferencias en el rendimiento académico de todas las dimensiones comunicativas en función del sexo. Estas discrepancias corroboran mejores valores de las niñas, todos ellos con una significación superior a $p<.01$ con la excepción del ítem distraerse que los niños tienen la media con mayor valor (2.19) que las niñas (2.14) y una significación de p<.001, aunque la diferencia es mínima, esta variable se presenta de forma negativa ya que en este valor es contrario, mostrando que estos tienen mayor facilidad para estar atentos y concentrados. En las diferencias de medias de cada grupo, las dimensiones seguir el hilo de una narración (3.45 $3.17=.28 ; \mathrm{p}<.01)$, describir una foto $(3.49-3.29=.20 ; \mathrm{p}<.01)$, terminar cuentos $(3.36-3.16=$ $.20 ; \mathrm{p}<.01)$ y escuchar cuentos $(3.53-3.31=.22, \mathrm{p}<.001)$ presentas los valores más altos. Igualmente, el contraste de estas variables muestra valores más elevados de $F>(3,84)$. 
Tabla 1.

Rendimiento lingüístico según el sexo

\begin{tabular}{lccc}
\hline & \multicolumn{3}{c}{ Media } \\
\cline { 2 - 4 } & Niña & Niño & F \\
\hline Escucha cuentos & 3.53 & 3.31 & $9,211^{* *}$ \\
Se distrae & 2.14 & 2.19 & $9,214^{* *}$ \\
Sigue narración & 3.45 & 3.17 & $13,148^{* * *}$ \\
Vocabulario preciso & 3.44 & 3.27 & $5,026^{*}$ \\
Termina cuentos & 3.36 & 3.16 & $5,555^{*}$ \\
Describe fotos & 3.49 & 3.29 & $6,090^{*}$ \\
Relación dibujo palabra & 3.33 & 3.18 & $5,127^{*}$ \\
Dividir palabra sonido & 3.61 & 3.55 & $4,890^{*}$ \\
Vocales iguales & 3.39 & 3.20 & $5,907^{*}$ \\
\hline
\end{tabular}

*valores significativos $p<.05$; **valores muy significativos $p<.01$;

***valores altamente significativos $p<.001$.

A continuación, la tabla 2 engloba datos que de nuevo están relacionados con el primer objetivo, aunque en este caso las variables dependientes hacen referencia a la lengua materna de estos alumnos (castellano y darija). En este caso, todas las áreas comunicativas presentan mejores resultados en el alumnado con lengua materna castellana, mostrando una alta significación en la todas las dimensiones con un valor $p<.001$ menos de en las destrezas a la hora de diferenciar vocales iguales y capacidad de concentración que obtienen un valor de $p<.01$. La descripción de fotos (3.81), la precisión en el vocabulario (3.77) y la capacidad para finalizar cuentos (3.68) presentan los valores con mayor valor de media, todas ellas pertenecen al grupo de niños con lengua materna castellana. En cambio, los de lengua materna darija muestran los datos con menor valor asociados a la capacidad para discriminar sonido (2.88) y terminar cuentos (3.01), con la peculiaridad de que la variable se distrae es la que tiene la media más baja (2.14) y en este caso pertenece al castellanoparlante, aunque esta dimensión tiene una connotación positiva puesto que muestra que estos alumnos se distraen menos que los darijoparlantes.

Tabla 2.

Rendimiento lingüístico según la lengua materna

\begin{tabular}{lccc}
\hline \multirow{2}{*}{ Lengua materna } & \multicolumn{3}{c}{ Media } \\
\cline { 2 - 4 } & Castellan & Darija & $\mathrm{F}$ \\
\hline Escucha cuentos & 3.67 & 3.26 & $29,194^{* * * *}$ \\
Se distrae & 2.14 & 2.34 & $5,110^{*}$ \\
Sigue narración & 3.60 & 3.12 & $36,038^{* * *}$ \\
Vocabulario preciso & 3.77 & 3.12 & $36,144^{* * *}$ \\
Termina cuentos & 3.68 & 3,01 & $60,602^{* * *}$ \\
Describe fotos & 3.81 & 3.15 & $75.639^{* * *}$ \\
Relación dibujo palabra & 3.57 & 3.06 & $33,866^{* * *}$ \\
Dividir palabra sonido & 3.63 & 3.09 & $37,182^{* * *}$ \\
Vocales iguales & 3.50 & 2.88 & $4.129^{*}$ \\
\hline nificativos $p<.05 ;$ **valores & muy significativos $p<.01 ; * * *$ valores altamente \\
$p<.001$. & \multicolumn{4}{l}{}
\end{tabular}


Una vez representados los datos que definen las diferencias de estos alumnos en función del sexo o la lengua materna, y con la intención de concretar el segundo objetivo, se presenta la tabla 3 donde los grupos se dividen en niñas de lengua materna darija y niños de lengua materna castellana. Podemos observar como la tendencia del sexo en el rendimiento académico comunicativo de los resultados anterior se invierte. De tal manera que en todas las dimensiones lingüísticas obtienen mejores resultados los niños de lengua materna castellana, destacando escucha cuento (3.66), la descripción de fotos (3.61) y la precisión en el vocabulario (3.56). En cambio, las medias con valores más pequeños están agrupadas en las variables distingue el sonido de vocales iguales (2.98), relación dibujo palabra (3.12) y la capacidad para estar atenta (2.15), siendo esta última la que menor valor tiene, aunque según lo explicado en los resultados anteriores el valor negativo de esta presenta una interpretación favorable para la valoración de la concentración y la atención de estas niñas. A pesar de que todas las variables presentan unos valores superiores al baremo mínimo de $\mathrm{F}(3,843)$ que muestra significancia, en este caso, la mayoría obtiene unos valores de $p<.01$ con la excepción de describir fotos y la capacidad de relacionar dibujos con palabras que presentan una significación de $p<.001$.

Tabla 3.

Rendimiento lingüístico niña de lengua materna darija y niño lengua materna castellana

\begin{tabular}{lccc}
\hline & \multicolumn{3}{c}{ Media } \\
\cline { 2 - 4 } & $\begin{array}{c}\mathrm{Ni} \\
\text { ña } \\
d a\end{array}$ & $\begin{array}{c}\text { Niño } \\
\text { castellano }\end{array}$ & $\mathrm{F}$ \\
& rija & & \\
\hline Escucha cuentos & 3.36 & 3.66 & $9,112^{* *}$ \\
Se distrae & 2.15 & 2.32 & $3,918^{*}$ \\
Sigue narración & 3.28 & 3.49 & $3,890^{*}$ \\
Vocabulario preciso & 3.26 & 3.56 & $7,628^{*}$ \\
Termina cuentos & 3.17 & 3.49 & $7,245^{*}$ \\
Describe fotos & 3.26 & 3.61 & $9,542^{* *}$ \\
Relación dibujo & 3.12 & 3.47 & $10,079^{* *}$ \\
palabra & & & \\
Dividir palabra & 3.25 & 3.49 & $4,540^{*}$ \\
sonido & & & \\
Vocales iguales & 2.98 & 3.30 & $5,837^{*}$ \\
\hline
\end{tabular}

*valores significativos $p<.05 ; * *$ valores muy significativos $p<.01 ; * * *$ valores altamente significativos $p<.001$.

Para completar los objetivos y centrados en el tercero, decidimos realizar un análisis de regresión logística binaria. Para ello, hemos seleccionado las variables de dimensiones lingüísticas que presentan mayor relevancia para nuestro estudio según la lengua materna de estos alumnos $(\mathrm{CO} 1=$ escucha cuentos; $\mathrm{CO} 2=$ se distrae; $\mathrm{CO} 3=$ sigue narración; $\mathrm{EO} 1=$ vocabulario preciso; $\mathrm{EO} 4=$ termina cuentos; $\mathrm{EO} 5=$ describe fotos; $\mathrm{EE} 3=$ relación dibujo palabra; $\mathrm{CF} 1=$ dividir palabra sonido; $\mathrm{CF}=$ vocales iguales).

Tabla 4.

Pruebas ómnibus de coeficientes de modelo

\begin{tabular}{lrrrr}
\hline & Chi-cuadrado & gl & & \multicolumn{1}{c}{ Sig. } \\
\hline Paso & 143,726 & & 9 &, 000 \\
Bloque & 143,726 & & 9 &, 000 \\
Modelo & 143,726 & & 9 &, 000 \\
\hline
\end{tabular}


El nivel de significación óptimo > 0.5 que muestra este modelo (tabla 4) permite obtener un pronóstico en relación con lengua materna castellana sobre la lengua materna darija. El nivel observado se distribuye con un p-valor $<(0,05)=0,00$ con un elevado valor chicuadrado 143,726 .

Tabla 5.

Clasificación variables dependientes

\begin{tabular}{llrrr}
\hline & & \multicolumn{3}{c}{ Pronosticado } \\
\cline { 3 - 5 } Observado & & \multicolumn{2}{c}{ Lengua materna } & Porcentaje \\
Español & Darija & correcto \\
\hline Lengua materna & Español & 161 & 257 & 38,5 \\
& Darija & 118 & 536 & 82,0 \\
Porcentaje global & & & & 65,0 \\
\hline
\end{tabular}

En este caso, el modelo de ecuación (ver tabla 5) logística valoró un porcentaje de predicción global para la lengua materna castellana $38.5 \%$ y un $82 \%$ para la lengua materna darija, datos estadísticamente significativos con un $65,45 \%$ de acierto. Resultados que indican que predice mejor el caso de los alumnos darijoparlantes. De ahí que las dos categorías de nuestro estudio combinadas en ecuación han pronosticado mejor a los alumnos de lengua materna darija.

Tabla 6.

Variables en la ecuación

\begin{tabular}{lrrrrrr}
\cline { 2 - 7 } & \multicolumn{7}{c}{$\begin{array}{c}\text { Error } \\
\text { estándar }\end{array}$} & \multicolumn{1}{c}{ Wald } & Gl & \multicolumn{1}{c}{ Sig. } & \multicolumn{1}{c}{ Exp(B) } \\
\hline Escucha cuentos &,- 237 &, 134 & 3,120 & 1 &, 077 &, 789 \\
Se distrae &,- 059 &, 069 &, 741 & 1 &, 389 &, 943 \\
Seguir y recordar narración &, 180 &, 139 & 1,680 & 1 &, 195 & 1,197 \\
Vocabulario preciso &,- 756 &, 148 & 26,158 & 1 &, 000 &, 470 \\
Termina cuentos &, 276 &, 147 & 3,543 & 1 &, 060 & 1,318 \\
Describir fotos &,- 460 &, 160 & 8,253 & 1 &, 004 &, 631 \\
Relación dibujo palabra &,- 227 &, 111 & 4,152 & 1 &, 042 &, 797 \\
Dividir palabra sonido &,- 110 &, 116 &, 896 & 1 &, 344 &, 896 \\
Vocales iguales &, 210 &, 094 & 5,027 & 1 &, 025 & 1,234 \\
Constante & 4,617 &, 525 & 77,359 & 1 &, 000 & 101,210 \\
\hline
\end{tabular}

Finalmente, y centrados en el tercer objetivo, los datos de las variables de la ecuación (tabla 6) muestran que existen áreas con significación en función de la lengua materna, destacando de la siguiente forma el orden de mayor significancia de la variable vocabulario preciso .000 (EO5), seguida de la descripción de fotos .004 (EO5), vocales iguales .025 (CF2) y relación dibujo palabra .042 (EE3).

\section{Discusión y conclusiones}

Según los datos aportados podemos considerar que existe un mejor rendimiento de las niñas en las áreas lingüísticas (Moss \& Brown, 1979) que corroboran una relación entre el mejor nivel de rendimiento de estas áreas y el sexo femenino (Harris, 1977; Maccoby \& Jacklins, 1974). Esto se traduce en valores superiores en el desarrollo de destrezas que potencian el dominio de la lengua que se refleja en la fluidez y precisión del vocabulario (Smolak, 1986). Diferencias que, a su vez, ponen de manifiesto niveles superiores en la comprensión y la expresión oral en función del sexo, en este caso a favor de las niñas. Esta regularidad en el vocabulario les facilita la construcción verbal de sus ideas y la capacidad de seguir el hilo de una conversación. Por todo esto, podemos admitir un desarrollo lingüístico superior en las niñas (Harris, 1977; 
Kimura, 1999). Del mismo modo, en conciencia fonética las niñas vuelven a obtener mejores resultados a la hora de diferenciar las distintas relaciones entre los sonidos y las palabras, además de reconocer y descodificar las asociaciones que se pueden dar entre ellas. De hecho, para Defior (2014) es un área que considerar a la hora de predecir futuras dificultades en la lectura.

Seguidamente, la valoración del rendimiento académico de las dimensiones comunicativas arroja datos que verifican la influencia de la lengua materna en el rendimiento de estos alumnos. De tal modo que en todas las áreas obtienen mejores resultados los castellanosparlantes, mostrando un nivel más alto en aquellas dimensiones que son elementales en este proceso de transición lingüística y educativa. En el caso del alumnado de lengua materna darija estas carencias se traducen en una expresión escrita y una conciencia fonética con un desarrollo inferior. Una situación que plantea como el lenguaje se puede convertir en el eje principal del proceso educativo y la evolución académica de estos alumnos (Palau, 2005).

Los datos anteriores toman aun mayor consistencia en la comparación de los grupos híbridos de niñas darijoparlante y niños castellanoparlantes en las que se invierte la tendencia de los resultados de este alumnado en función del sexo. En este caso, la determinación de la lengua materna como base principal del rendimiento académico provoca que los resultados se transformen, mostrando mejores valores en los niños. Datos que revelan que la lengua tiene mayor peso que el sexo, traducido en mejores resultados de todas las dimensiones comunicativas de los niños castellanoparlantes. Efectos que nos hacen plantear la asociación del sexo a factores individuales y la lengua a factores externos claramente definidos por las peculiaridades que nos plantean la diversidad del contexto (Vygotsky, 1962). Es por ello por lo que la lengua materna es la que marca las diferencias entre estos dos grupos prevaleciendo por encima del sexo. Moerk (1975) defiende que la lengua materna sostiene todos los elementos para enseñar a los niños el lenguaje gramatical, en cambio, otros solo le asignan una influencia superficial (Gleitman, Newporty \& Gleitman, 1984).

Sea como fuere, las evidencias obtenidas en esta investigación permiten considerar que los alumnos de lengua materna castellana muestran mejor calidad, precisión e incremento de vocabulario que favorece la expresión de sus pensamientos, además de potenciar la integración en el medio social (Méndez, Barrientos, Macías y Peña, 2006). Estas diferencias también se muestran en la expresión escrita consolidando mejores habilidades a la hora de utilizar un proceso abstracto que, junto a la discriminación de sonidos, complementan un proceso encaminado a utilizar nuevos canales que permitirán avanzar en el desarrollo de la lectoescritura a través de la utilización simultánea de un código oral y otro escrito. De ahí la significación e importancia, al ser complementaria, de las mejores destrezas de los castellanoparlantes en la conciencia fonética. De hecho, la principal manifestación de esta diferencia entre estos dos grupos se encuentra en el sistema vocálico, en el caso del castellano cinco ( $\mathrm{a}, \mathrm{e}, \mathrm{i}, \mathrm{o}, \mathrm{u})$ y el árabe tres $(\mathrm{i}, \mathrm{a}, \mathrm{u})$, los alumnos darijoparlantes encuentran dificultades en la reproducción de sonidos vocálicos (Abu-Shams, 2011). Del mismo modo que comentamos anteriormente, los datos negativos de la variable estar atentos a los castellanoparlantes les favorece en la consecución y el recuerdo de los argumentos que se presenta ante una conversación, permitiéndoles avanzar en el esquema completo del relato en el momento de su repetición.

Partiendo de la influencia de la lengua materna en el proceso lingüístico de estos alumnos podemos afirmar que esta se presenta como el elemento principal que interfiere en el avance académico de los alumnos con una lengua materna diferente a la oficial y que, además, en este caso, carece de estructura formal, quedando privada de su equivalencia a una forma semipresencial. De esta forma, se destaca la debilidad en áreas comunicativas relacionadas con la expresión oral, la conciencia fonética y la expresión escrita que tienen como indicador principal las carencias iniciales en la precisión del vocabulario de los alumnos con lengua materna darija. Unas áreas que muestran las diferencias entre los alumnos castellanoparlantes y 
los darijo parlantes a la vez que nos proyecta las futuras repercusiones en el progreso lingüístico de estos alumnos. Un avance que les permitirá concretar la consecución de habilidades asociadas con la integración entre el input acústico, el conocimiento lingüístico, el conocimiento del mundo y el contexto comunicativo (Olmedo, 2017), en este caso, definidas por las carencias de la expresión oral de los darijoparlantes. Igualmente, esta debilidad se muestra en la expresión escrita, que requiere el dominio visual y auditivo, estos dos claramente relacionados con la conciencia fonética, en las que están implicadas la discriminación de los sonidos y su relación con las letras. Todo ello les va a facilitar u obstaculizar la transición de lo concreto a lo abstracto que tiene como principal función la ejecución de la lectoescritura. Un acto fundamental en el desarrollo de estos alumnos y este periodo, que se establece como principal indicador del nivel de estos niños y predice el rendimiento futuro del alumnado.

En definitiva, los análisis de los datos responden a cuestiones centradas en el peso que tienen en el rendimiento académico el sexo o el lenguaje, poniéndose de manifiesto, en este estudio, que la lengua materna se convierte en la principal causa de las diferencias en el rendimiento lingüístico de estos alumnos. Una situación que plantea la importancia de las competencias comunicativas como medio elemental para el progreso académico.

En el desarrollo de este trabajo la edad de la muestra de los alumnos, demasiado jóvenes para la cumplimentación del instrumento, situación que nos obligó a que la elaboración del mismo fuera adaptada para su formulación por parte los docentes, se convirtió en la principal limitación de nuestra investigación.

\section{Referencias}

Abu-Shams, L. (2011). Prestamos e influencias léxicas en la cultura alimentaria magrebí. Zainak, 34, 413-426. Recuperado de http://hedatuz.euskomedia.org/8463/1/34413426.pdf

Bartolomé, M. (2017). Diversidad educativa ¿Un potencial desconocido? Revista de Investigación Educativa, 35 (1), 15-33. doi: https://doi.org/10.6018/rie.35.1.275031

Bowlby, J. (1993). El vínculo afectivo. Barcelona: Paidós.

Chomsky, A. (1968). El lenguaje y el entendimiento. Barcelona: Planeta Agostini.

Defior, S. (2014). Procesos implicados en el reconocimiento de las palabras escritas. Aula: revista de enseñanza e investigación educativa, 20, 25-44. Recuperado de http://campus.usal.es/ revistas_trabajo/index.php/0214-3402/article/view/12560

Gay, G. (2000). Culturally responsive teaching. New York: Teachers College Press.

Glass, G., \& Stanley, J. (1980). Métodos estadísticos aplicados a las Ciencias Sociales. Madrid: Prentice-Hall Internacional.

Gleitman, L., Newport, M., \& Gleitman, H. (1984). The current status of the motherese hypothesis. Journal of child language II, 11, 43-79. https://doi.org/10.1017/S0305000900005584

Goleman, D. (1996). Inteligencia emocional. Barcelona: Kairós.

Gómez, D., Oviedo, R., \& Martínez, I. (2011). Factores que influyen en el rendimiento académico de estudiantes universitarios. Educación y humanidades, 5(2), 90-97. Recuperado de http://tecnociencia.uach.mx/numeros/v5n2/data/Factores_que_influyen_en_el_ rendimiento_academico_del_estudiante_universitario.pdf

González, M. J., \& Martín, I. (2017). Effects on Reading of an Early Intervention Program for Spanish Children at Risk of Learning Difficulties. Remedial and Special Education. 8(2), 67-75. doi: https://doi.org/10.1177/0741932516657652

Goñi, E., Ros, I., \& Fernández, O. (2018). Academic performance and school engagement among secondary school students in accordance with place of birth, gender and age. European Journal of Education and Psychology, 11(2), 93-105. https://doi.org/10.30552/ejep.v11i2.224 
Harris, L. J. (1977). Sex differences in the growth and use of language. In E. Donelson \& J. Fullarhorn (Eds), Women: a psychological perspective (pp. 79-94). New York: Wiley.

Harris, J. L., Kamhi, A. G., \& Pollock, K. E. (Eds.). (2001). Literacy in African American communities. Mahwah: Lawrence Erlbaum Associates.

Kimura, D. (1999). Sex and cognition. Massachusetts: MIT Press. doi: https://doi.org/10.7551/mitpress/6194.001.0001

Lamas, H. (2015). Sobre el rendimiento escolar. Propósitos y Representaciones, 3(1), 313-386. doi: https://doi.org/10.20511/pyr2015.v3n1.74

Latorre, M. J., Mateos, F., Olmedo; F. J., \& Esteban, M. (2018). Modelos culturales en un contexto multicultural. International Journal of Educational Research and Innovation, (9), 31-35. Recuperado de https://www.upo.es/revistas/index.php/IJERI/article/view/2669

Maccoby, S., \& Jacklins, C. (1974). The psychology of sex differences. California: Stanford University Press.

Maier, H. (2000). Tres teorías sobre el desarrollo del niño: Erikson, Piaget y Sears (10 ed.). Buenos Aires: Amorroutu.

Marjoribanks, K. (2005). Family and Children's Outcomes. Educational Psychology, 25(6), 647-657. doi: https://doi.org/10.1080/01443410500344704

Maroco, J., \& García, T. (2013). Qual a fiabilidade do alfa de Cronbach? Questões antigas e soluções modernas? Laboratório de Psicologia, 4 (1), 65-90. doi: https://doi.org/10.14417/lp.763

Mateos, F., \& Olmedo, F. J. (2018). Patente $N^{o}$ : 00/2018/1429. Madrid, España: Registro General de la Propiedad Intelectual.

Mateos, F., Olmedo, F. J., Esteban, M., \& Amador, L. A. (2019). Lengua materna, cultura y rendimiento en un contexto multicultural de Educación Infantil. Ocnos, 18(2), 44-54. Doi: https://doi.org/10.18239/ocnos_2019.18.2.1966

Méndez, L., Barrientos, E., Macías, N. y Peña, J. (2006). Manual práctico: Desarrollo de la segunda infancia. México: Trillas.

Moerk, E. (1975). Verbal interactions betwen children and their mothers during the preschool years. Development Psychology, 11, 788-795. doi: https://doi.org/10.1037/00121649.11.6.788

Mora, F. (2013). Neuroeducación. Madrid: Alianza Editorial.

Moss, J. D. \& Brown, F. G. (1979). Sex bias and academic perfomance: An empirical study. Journal of Educational Measurement, 3, 197-201. doi: https://doi.org/10.1111/j.17453984.1979.tb00101.x

Olmedo, F.J. (2017). Cultura y Rendimiento del Alumnado de Segundo Ciclo de Educación Infantil en un Contexto Multicultural (Tesis Doctoral). Universidad de Granada, Granada.

Palau, E. (2005). Aspectos básicos del desarrollo infantil: la etapa de 0 a 6 años. Barcelona: CEAC.

Piaget, J. (1988). Psicología evolutiva de Jean Piaget. México: Paidós.

Rodríguez, R.M. (2017). Cultura escolar vs cultura familiar: vincular escuela, familia y comunidad desde la pedagogía culturalmente responsiva. Interacções, 43, 7-27. Doi: https://doi.org/10.25755/int.12029

Roid, H., \& Sampers, J. (2011). Merrill-Palmer-Revised Scales of Development. EE.UU: Stoelting Company.

Smolak, L. (1986). Infancy. Englewood cliffs, NJ: Prentice Hall.

Sanhueza, S. V. y Cardona, C. (2009). Evaluación de la sensibilidad intercultural en alumnado de Educación Primaria escolarizado en aulas culturalmente diversas. Revista de Investigación Educativa, 27(1), 247-262. Recuperado de: https://revistas.um.es/rie/article/view/94391/103021

Sousa, D.A. (Ed.). (2014). Neurociencia educativa. Madrid: Narcea.

Tejedor, F. J. y García-Valcarcel, A. (2006). Causas del bajo rendimiento del estudiante universitario (en opinión de los profesores y alumnos). Propuestas de mejora en el 
marco del EEES. Revista de Educación, 342, 443-473. Recuperado de http://www.revistaeducacion.mec.es/re342/re342_21.pdf

Turner, M. \& Cross, R. (2016). Making space for multilingualism in Australian schooling. Language and Education, 30 (4), 289-297. doi: https://doi.org/10.1080/09500782.2015.1114627

Vygotsky, L.S. (1962). Thought and language.Cambridge, M.A: MIT Press. doi: https://doi.org/10.1037/11193-000

(1996). A farmação social da mente: o desenvolviendo dos procesos superiores. São Paulo: Martins Fontes.

Wild, R. (2011). Etapas del desarrollo. Barcelona: Herder. doi: https://doi.org/10.2307/j.ctvt9jzs6 